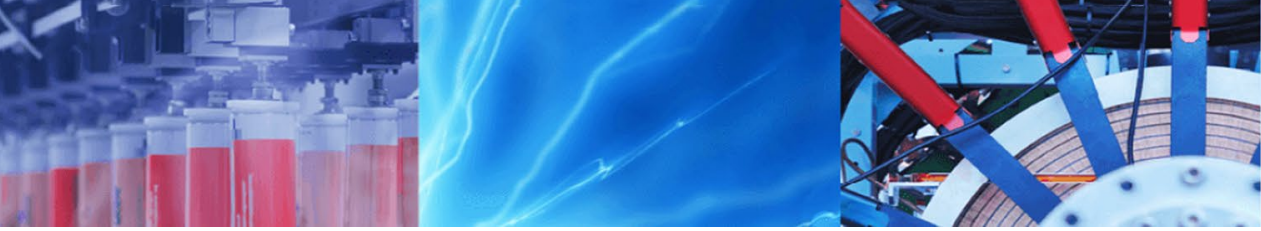

\title{
Microscopic studies on the polymers decomposition in a closed volume at elevated temperatures in the presence of bulk $\mathrm{NiCr}$ alloy
}

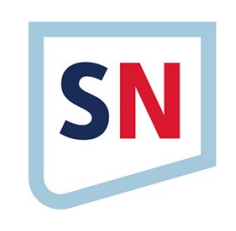

\author{
Roman M. Kenzhin ${ }^{1}$ - Yuri I. Bauman ${ }^{1}$ - Alexander M. Volodin ${ }^{1} \cdot$ Ilya V. Mishakov ${ }^{1,2}$ (I) $\cdot$ Vladimir I. Zaikovskii $^{1}$ (D) \\ Aleksey A. Vedyagin ${ }^{1,2}$ (i)
}

(c) Springer Nature Switzerland AG 2018

\begin{abstract}
In the present work, self-disintegration of the bulk metallic $\mathrm{Ni}$-Cr alloy in the presence of different polymer compounds under the conditions of closed volume and elevated temperatures is studied. It was found that in the cases of polymers containing in addition to carbon only hydrogen atoms (polyethylene) or only halogen atoms (polytetrafluoroethylene), self-disintegration does not proceed noticeably even at temperatures as high as $800^{\circ} \mathrm{C}$. The predominating form of the solid product is non-catalytic carbon shaped as microspheres or their sintered agglomerates. On the contrary, for decomposition of the polymer containing hydrogen and halogen atoms (polyvinylchloride), self-dispersion of the bulk alloy is significantly facilitated. This process is accompanied with formation of catalytically derived forms of structured carbon within all the studied temperature range $\left(500-800^{\circ} \mathrm{C}\right)$. The most common form of the carbon deposits is nanofibers. At the same time, some unusual carbon structures were observed, among which the solid-phase carbon-nickel microcrystals of a regular cubic shape with pyramidal cavities on each facet are of special interest. The studied process can be considered as an alternative approach for utilization and recycling of the plastic wastes.
\end{abstract}

Keywords Polymers decomposition $\cdot$ Closed volume $\cdot \mathrm{NiCr}$ alloy $\cdot \mathrm{RAPET} \cdot \mathrm{Nanostructured}$ carbon materials

\section{Introduction}

Nowadays carbon nanofibers (CNF) attract a great attention due to their enhanced thermal, electric and mechanical properties [1-3]. Most of commercial technologies to obtain the CNFs imply chemical vapor deposition in various alternatives including the catalytic one. As it is well known, the catalytic properties in the catalytic chemical vapor deposition (CCVD) are performed by metals of iron group ( $\mathrm{Fe}, \mathrm{Co}, \mathrm{Ni})$ and their alloys supported on the surface of the carrier with developed surface area. On the other hand, recently we have demonstrated the possibility to use bulk metallic nickel or its alloys in a bulk state as a catalyst for the synthesis of CNFs from halogenated hydrocarbons [4-6]. During the interaction of such aggressive organic medium with bulk metal items the latter undergo self-disintegration with formation of nickel particles with uniform size of $200-250 \mathrm{~nm}$. The particles formed exhibit excellent activity in CNFs growth. In order to obtain possible solid-phase intermediates, we have performed experiments on detail studies of the mentioned interaction at elevated temperatures in closed volume (autoclave conditions), when the gaseous products withdrawal is practically absent $[7,8]$. A similar approach is well known in the literature to be successfully applied for the synthesis of various carbon and other inorganic materials [9-11]. The described process was named as reactions under autogenic pressure at elevated temperature (RAPET). As it was found in our recent studies, the interaction of metallic nickel with halogen-substituted hydrocarbons (for example, hexafluorobenzene) in closed volume leads to appearance of solid phase structures,

$\triangle$ Aleksey A. Vedyagin, vedyagin@catalysis.ru| ${ }^{1}$ Boreskov Institute of Catalysis SB RAS, Novosibirsk, Russian Federation 630090. ${ }^{2}$ Tomsk Polytechnic University, Tomsk, Russian Federation 634050.

SN Applied Sciences (2019) 1:139 | https://doi.org/10.1007/s42452-018-0152-2 
hypothetically - microcrystals of nickel halogenides. These compounds are metastable and undergo rapid destruction at high temperatures. This process is accompanied by formation of carbon nanofibers doped with a small amount of halogen, which is very important for their further application due to enhanced surface properties (for example, better hydrophily). Thereby, the use of halogen-containing compounds as a source for CNFs production can be also considered as a perspective approach for processing of different organochlorine wastes into valuable carbonaceous product.

Besides the processing of organochlorine wastes, utilization of spent polymeric items (solid domestic wastes, plastics, etc.) is of great importance, since the world production of such items grows year by year. As a rule, industrial synthetic polymers are quite stable chemical compounds. Most of them (for instance, polyethylene) are resistant towards action of sun light and air oxygen, and do not undergo self-destruction for a long period of time. Among polymeric compounds, the halogen-containing ones (like polyvinylchloride, fluoroplastic) are the most dangerous. Their combustion in air leads to formation of variety of gaseous toxic compounds. One of the perspective approaches of polymers utilization known in the literature is their processing into various carbon materials (for example, carbon nanotubes) [12-15]. As a rule, the catalyst is represented by transition metal or by salt of such metal. In some cases, the catalyst can be added into reactor in a gas phase [for example, $\mathrm{Fe}(\mathrm{CO})_{5}$ ] [16].

In the present research, an approach for processing of polymeric wastes (polyethylene, polyvinylchloride, Teflon) in the presence of bulk $\mathrm{NiCr}$ alloy self-disintegrated under the action of aggressive reaction medium with formation of uniform nickel particles catalyzing the growth of CNFs is suggested. The possibility to perform such a process in closed volume at elevated temperature was studied. The attractive advantages of this approach are that it goes via one stage and looks very simple in terms of technical realization.

\section{Experimental}

Commercial Ni-Cr alloy (nichrome; $80 \mathrm{wt} \% \mathrm{Ni}, 20 \mathrm{wt} \% \mathrm{Cr}$ ) in the shape of wire of $0.1 \mathrm{~mm}$ in diameter was used as a bulk metal item. The polymer samples to be decomposed were polyethylene of low pressure (household plastic bag), polyvinylchloride (insulating braid), and polytetrafluorethylene (Teflon tape).

Experiments on polymer decomposition in a close volume were performed using quartz ampoules $(\mathrm{d}=4-5 \mathrm{~mm}$, $\mathrm{V} \sim 0.2 \mathrm{ml}$ ) as a reactor $[7,8]$. A piece of nichrome wire of about $0.2-0.3 \mathrm{mg}$ was placed in an ampoule together with
2-3 mg of polymer (polyethylene, Teflon or polyvinylchloride). The ampoule with reaction mixture was sealed and brought to thermal treatment at certain temperature for $2 \mathrm{~h}$. The accuracy of temperature measurements was $\pm 2{ }^{\circ} \mathrm{C}$.

After the reaction procedure, the ampoule was carefully opened for microscopic investigation. The obtained carbon material was studied by scanning electron microscopy (SEM) on a JSM-6460 (Jeol, Japan) electron microscope with a resolution of $4 \mathrm{~nm}$ in the range of magnifications from $5 \times$ to $300,000 \times$. The local energy-dispersion X-ray (EDX) microanalysis was carried out using EDAX spectrometer with energy resolution $127 \mathrm{eV}$.

HRTEM images were obtained using a JEM-2010 electron microscope (Jeol, Japan) with a lattice-fringe resolution of $0.14 \mathrm{~nm}$ at accelerating voltage of $200 \mathrm{kV}$. The samples for HRTEM were prepared on a perforated carbon film mounted on a copper grid.

\section{Results and discussion}

As it was reported earlier, self-disintegration of bulk metallic nickel and its alloys can be significantly facilitated when the reaction mixture contains both hydrogen and halogen sources $[7,8]$. In the case of organic compounds containing hydrogen or halogen only (for example, hexamethylbenzene or hexafluorobenzene) used as a carbon source, this process is rather complicated. Polyethylene, the most popular domestic polymer material and, therefore, the most common plastic waste, contains only carbon and hydrogen atoms. Thereby, taking into account the results of previous studies, it was expected that its catalytic decomposition accompanied with selfdisintegration of the bulk $\mathrm{NiCr}$ alloy in closed volume at elevated temperature will be hindered. In order to examine this expectation, the ampules with reagents (piece of $\mathrm{NiCr}$ wire and polyethylene) were consecutively calcined at temperature from 400 to $800{ }^{\circ} \mathrm{C}$ with a step of $100^{\circ} \mathrm{C}$. For the resulted contents we have tried to apply ferromagnetic resonance (FMR) method as described in [5-8]. This method was shown to be an efficient tool for investigation of nickel-containing samples. The most convenient case of its application is the study of nichrome, since this alloy does not have FMR signal in the initial state but appearance of particles of metallic nickel even in a small amount is well detectable. In the present study, it was found that FMR signal indicating the beginning of $\mathrm{NiCr}$ self-disintegration did not appear even for samples treated at $700^{\circ} \mathrm{C}$. Note that for the samples heated at higher temperature, the applicability of FMR method is restricted due to strong microwave absorption by carbon products resulting from thermal decomposition of the organic precursor. 

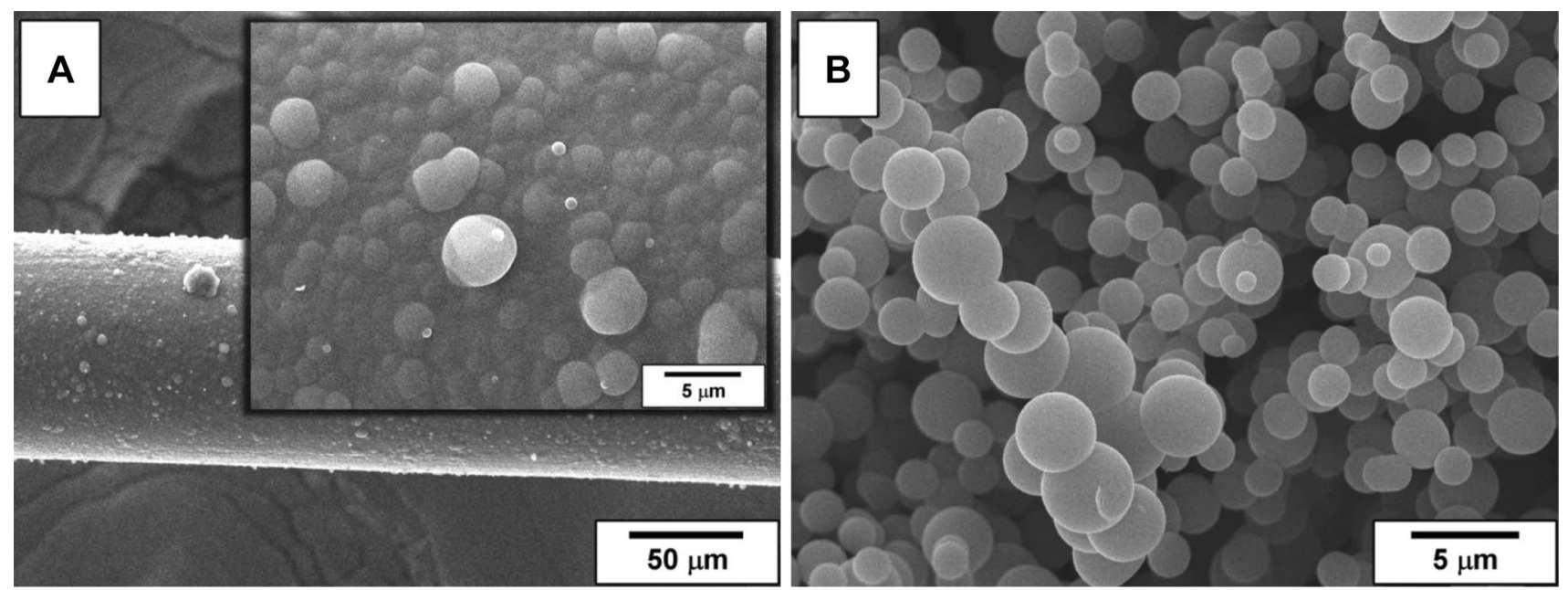

Fig. $1 \mathrm{SEM}$ image of $\mathrm{Ni}-\mathrm{Cr}$ wire after interaction with polyethylene at $800^{\circ} \mathrm{C}$ under the RAPET conditions (a) and carbon product obtained under the same conditions via pyrolysis of polyethylene without a catalyst (b)
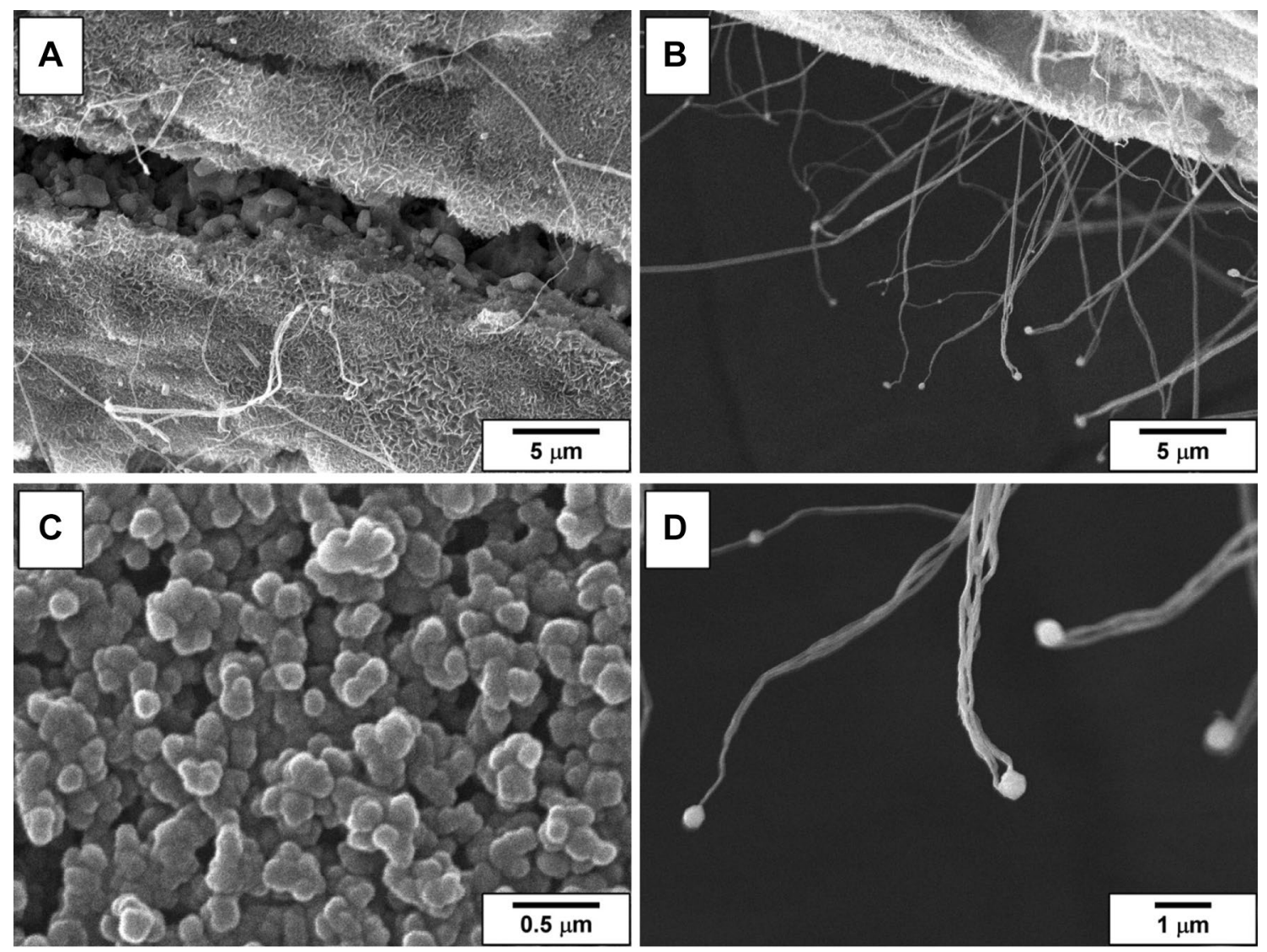

Fig. 2 SEM images of Ni-Cr wire after interaction with Teflon at $800^{\circ} \mathrm{C}$ under the RAPET conditions (a, b), and obtained carbon deposits (c, d)

Moreover, at reaction temperatures of $500{ }^{\circ} \mathrm{C}$ and above the appearance of an intensive singlet $\mathrm{g}=2.003$ typical for various carbon materials becomes noticeable $[7,8]$.
Figure 1a shows the micrographs of the nichrome sample after its interaction with polyethylene at temperature of $800^{\circ} \mathrm{C}$. As seen, the surface of nichrome wire is covered 

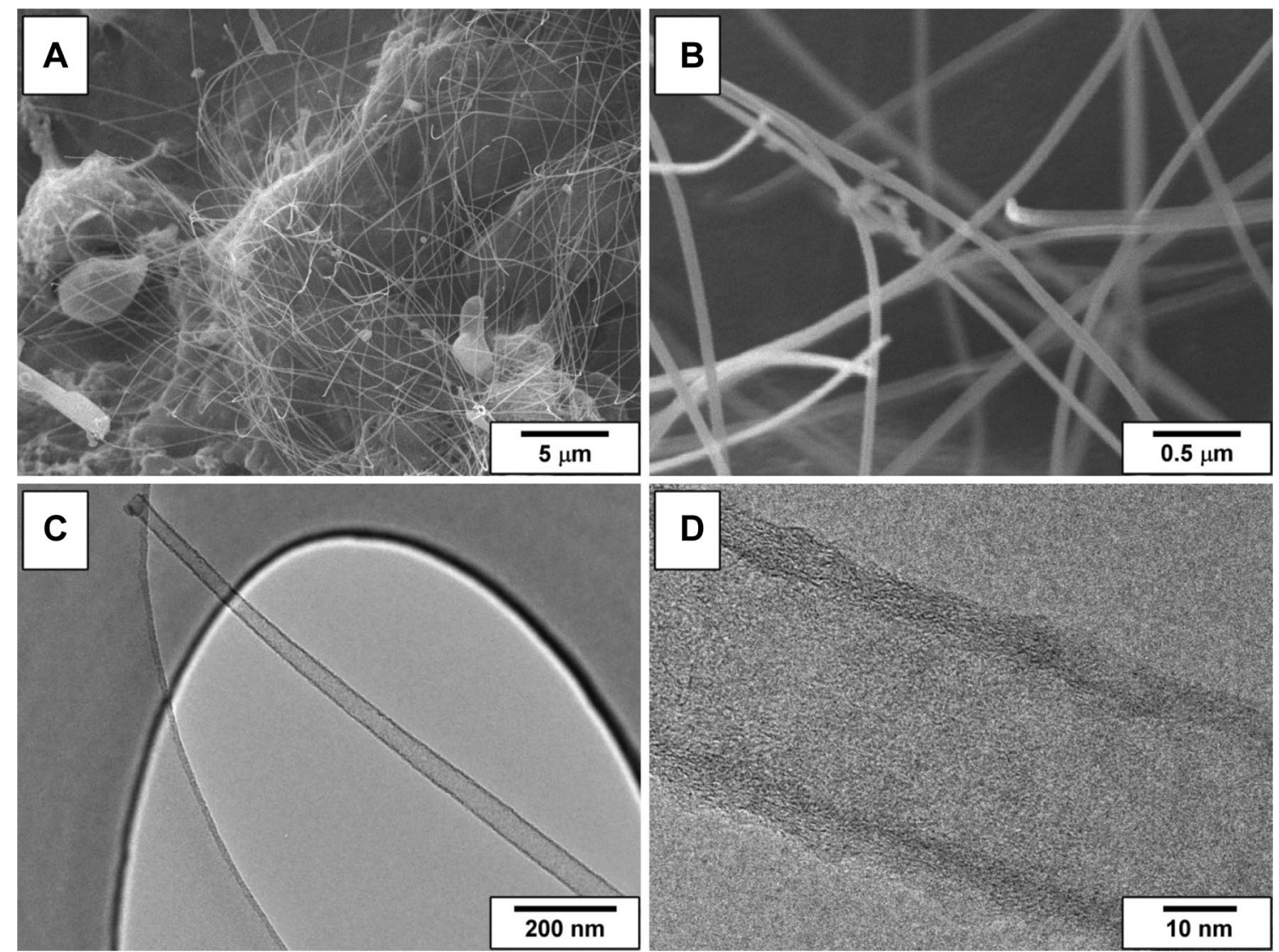

Fig. 3 SEM $(\mathbf{a}, \mathbf{b})$ and TEM $(\mathbf{c}, \mathbf{d})$ images of the carbon product resulting from Teflon decomposition at $800^{\circ} \mathrm{C}$ under RAPET conditions without adding a catalyst (nichrome)

with non-catalytic spherical carbon deposits, which are believed to be similar to the carbon reported in [8]. It is worth noting that analogous spherical carbon was also found on the ampule walls after pyrolysis of the organic substrate at a high temperature (Fig. 1b). Appearance of similar structures in the absence of a catalyst was recently reported by Pol [12]. Thereby, it was confirmed for the plastic source that self-disintegration of bulk $\mathrm{NiCr}$ alloy with the formation of carbon nanofibers does not take place even at temperatures as high as $800^{\circ} \mathrm{C}$. This observation is in good agreement with previously made assumption about the necessity of the presence of both the hydrogen and halogen source in the reaction medium $[7,8]$.

At the next stage, the opposite case was considered when the plastic source contains halogen only. The mentioned condition is suited by polytetrafluoroethylene (Teflon), which is also of a quite wide usage, in particular, for domestic applications. In the present case, similarly to the previous one, appearance of FMR signal at temperatures up to $500^{\circ} \mathrm{C}$ was not observed. Registration of FMR spectra at higher temperatures does not make sense, since the microwave adsorption by the sample becomes too strong. However, contrary to polyethylene, which is characterized by the presence of an intensive narrow singlet with $\mathrm{g}=2.003$ typical for variety of carbon materials $[7,8]$, formation on non-catalytic carbon from Teflon takes place at significantly higher temperatures. Micrographs of nichrome interaction with polytetrafluoroethylene at temperature of $800^{\circ} \mathrm{C}$ are presented in Fig. 2. As it is well seen from Fig. 2a, all the surface of nichrome wire is covered with a thick layer of nanostructured carbon material. At least two types of carbon deposits can be recognized from the images. First of all, it is dense layer of compacted carbon structures, which is well seen in Fig. 2a, b. Figure $2 c$ demonstrates the morphology of this layer with higher magnification. The layer is constructed from dense carbon deposits of $0.1 \mu \mathrm{m}$ in diameter, and a developed porous structure. These carbon deposits seem to be of noncatalytic origin. The second type of structured carbon is prolonged carbon fibers with a catalytic particle at the tip (Fig. 2b, d). In general, these fibers are of 10-50 nm in diameter and of 30-50 $\mu \mathrm{m}$ in length. Some of the particles are connected with several (from 3 to 6 ) carbon fibers twisted into a bunch (Fig. 2d). Among these two types of carbon, the dense non-catalytic deposits predominate in terms of weight. 

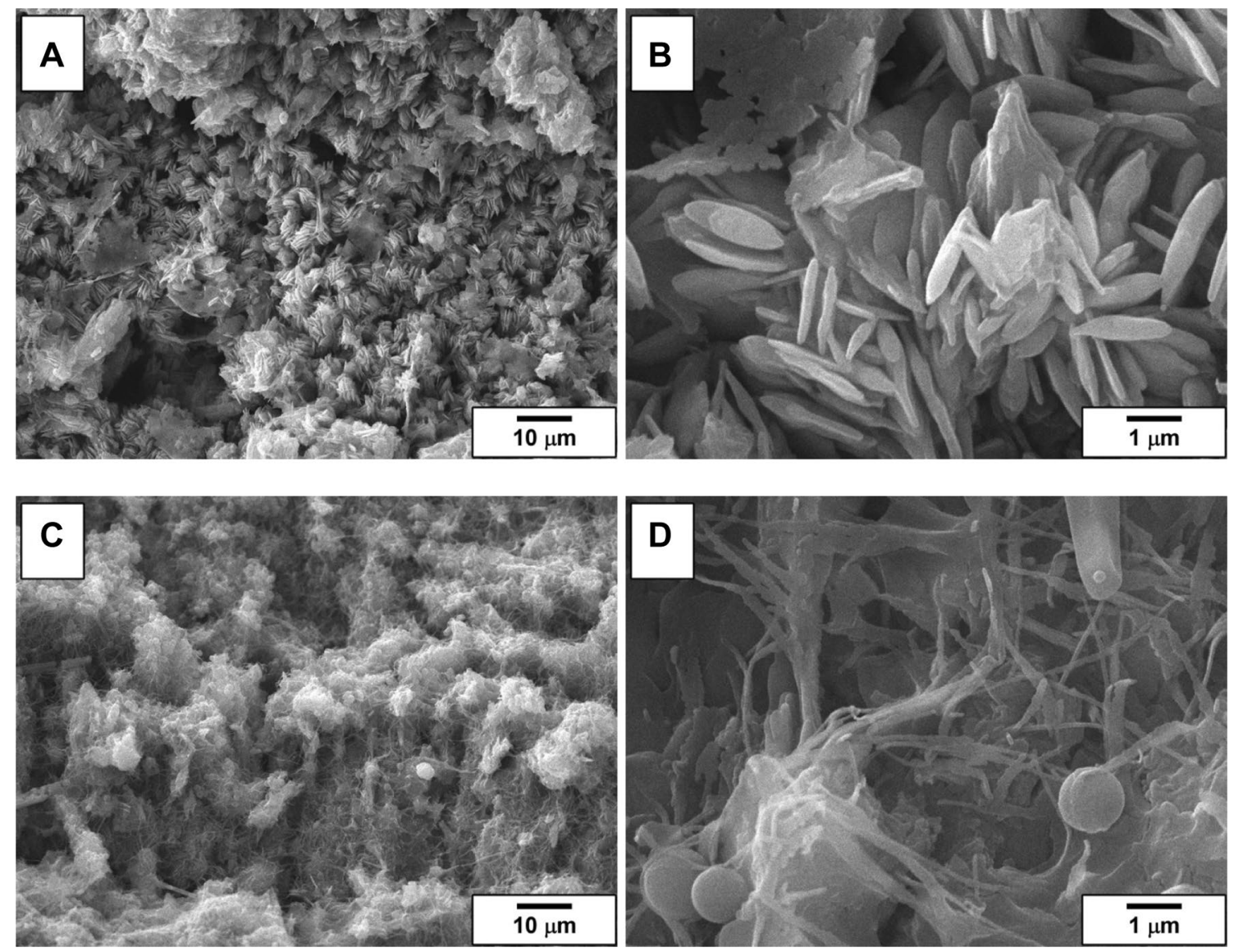

Fig. 4 SEM images of the carbon product obtained after interaction of $\mathrm{Ni}-\mathrm{Cr}$ wire with polyvinylchloride at $500{ }^{\circ} \mathrm{C}$ under RAPET conditions

Thereby, in the case of completely halogenated polymer (when all hydrogen atoms are substituted with halogen), self-disintegration of bulk nickel alloy is also complicated. Carbon nanofibers formed via catalytic route are present in a minor amount, while non-catalytic dense carbon deposits are found to be the predominating product.

On the other hand, it is worth noting that similar experiments performed in closed volume with Teflon at $800^{\circ} \mathrm{C}$ without using the catalyst (nichrome wire) led to the formation of non-catalytic carbon product of a fibrous shape (Fig. 3a, b). According to the TEM data (Fig. 3c, d), morphological structure of these deposits corresponds to multiwall nanotube. The diameter of these nanotubes is in the range of $20-40 \mathrm{~nm}$. The number of the carbon layers within the wall seems to be about 10 . The length of nanotubes is quite great, reaching $50-100 \mu \mathrm{m}$.

The last example of the studied polymeric substrates is polyvinylchloride. This representative of the plastics also has a great contribution into the national economy. Since it contains both the hydrogen and the halogen sources, its decomposition in close volume completes the performed study. Examination of this system by means of electron paramagnetic resonance revealed that contrary to polyethylene and polytetrafluoroethylene, formation of condensed carbonaceous structures takes place at relatively lower temperatures-already at $400^{\circ} \mathrm{C}$. At the same time, the bulk metal self-disintegration was not observed until temperatures above $600^{\circ} \mathrm{C}$ (FMR signal is absent). Similarly to the previous cases, no FMR signal was registered at higher temperatures due to strong microwave adsorption. On the other hand, since the formation of carbon deposits took place at lower temperatures, the product obtained was studied by SEM technique. Figure 4 demonstrates carbon structures observed after the interaction of nichrome with polyvinylchloride at $500^{\circ} \mathrm{C}$. The thick and fluffy carbon layer formed over the surface of $\mathrm{Ni}-\mathrm{Cr}$ wire is represented by two types of carbon structures. The chaotically agglomerated dense microdiscs of $1-2 \mu \mathrm{m}$ in size are shown in Fig. $4 \mathrm{a}, \mathrm{b}$. These structures are believed to have non-catalytic nature. The second type is a catalytically derived fibrous carbon presented in Fig. 4c, d. Very similar interlaced carbon nanofibers were reported recently to be formed during the catalytic decomposition of 1,2-dichloroethane over the same nichrome wire in a flow regime (an open reactor system) [4-6]. The currently obtained nanofibers are of tens $\mathrm{nm}$ in diameter and of 

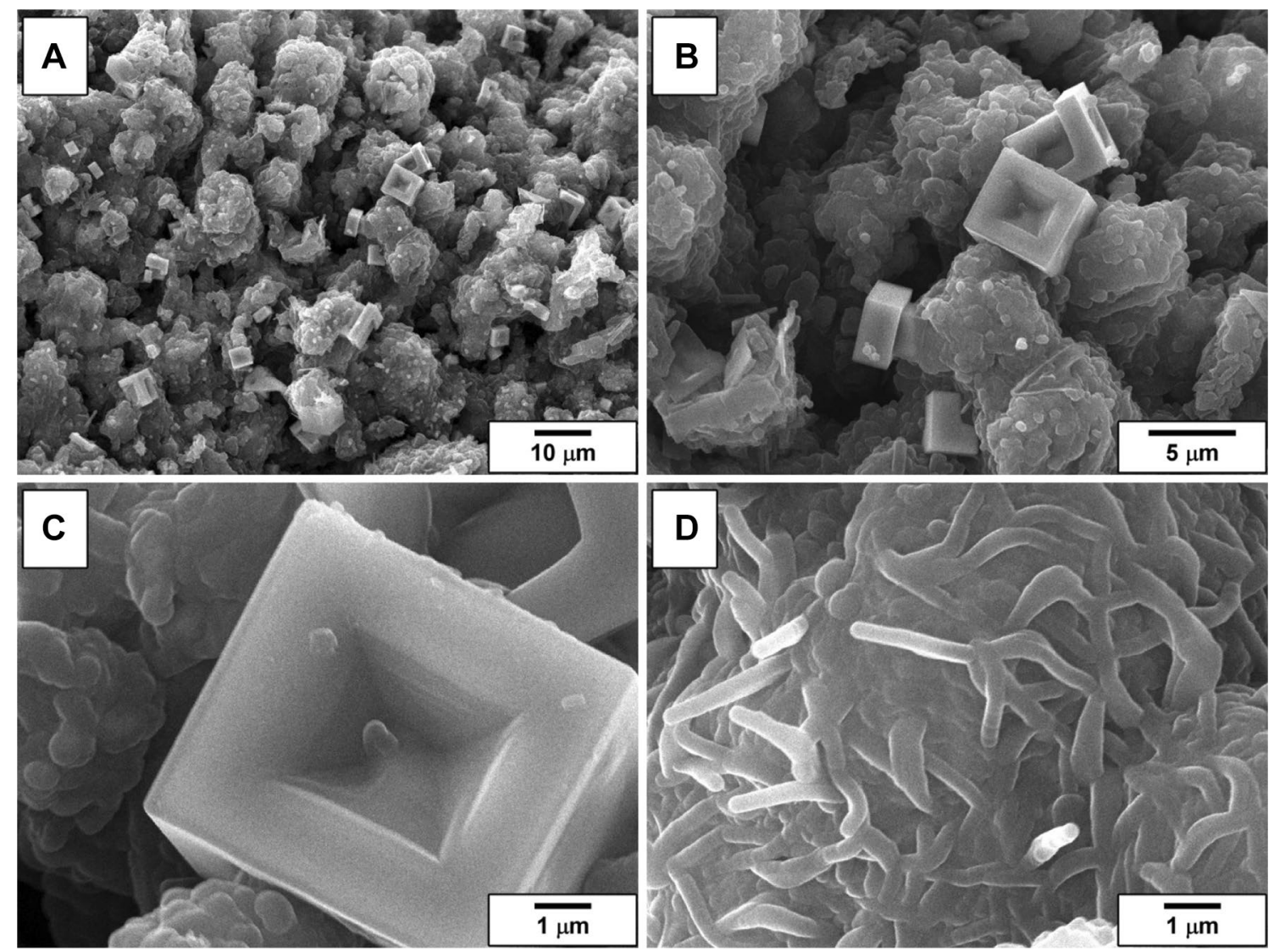

Fig. 5 SEM images of the carbon product obtained after interaction of $\mathrm{Ni}-\mathrm{Cr}$ wire with polyvinylchloride at $800{ }^{\circ} \mathrm{C}$ under RAPET conditions
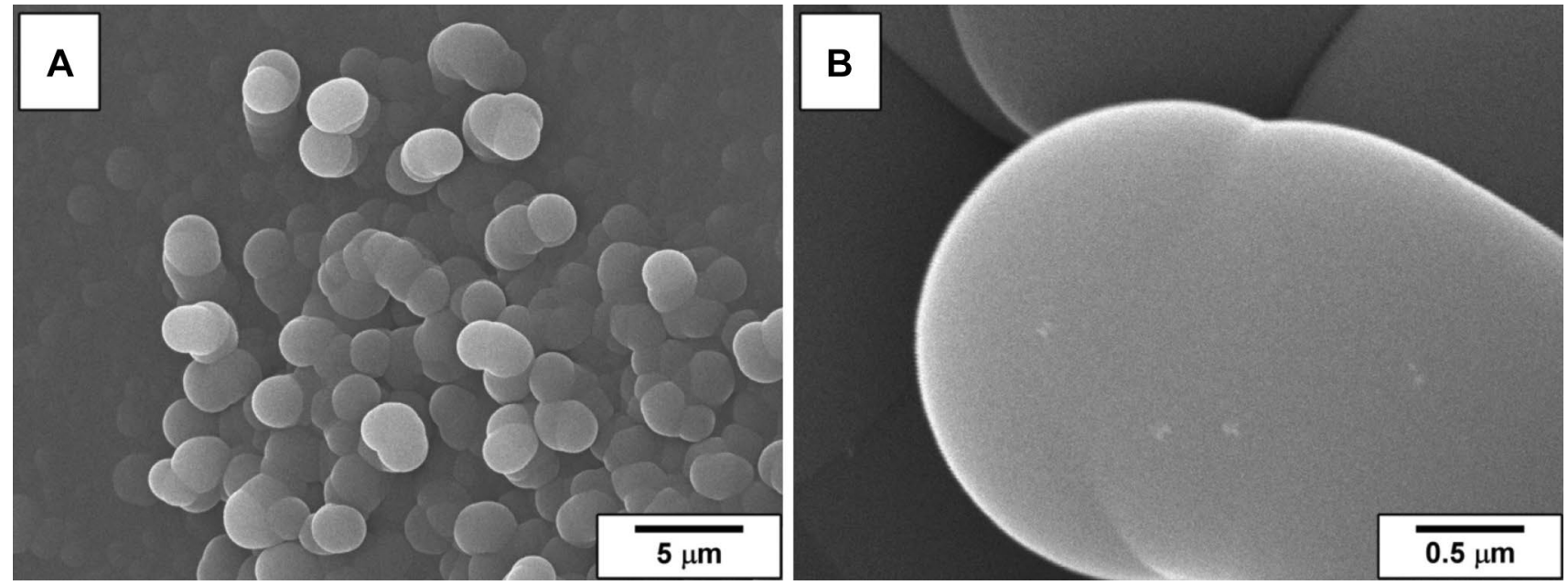

Fig. $6 \mathrm{SEM}$ images of the carbon product resulting from polyvinylchloride decomposition at $800^{\circ} \mathrm{C}$ under RAPET conditions without adding a catalyst (nichrome)

hundreds $\mathrm{nm}$ in length. It should be emphasized that all the formed carbon deposits are significantly functionalized with surface oxygen groups. According to the data of electron dispersive X-ray (EDX) analysis, they consist of about 80 at.\% of carbon and $10-20$ at.\% of oxygen. The residual components are chlorine, nickel (small particles catalyzing the carbon nanofibers growth), and chromium. Appearance of such a great amount of oxygen groups is

\section{SN Applied Sciences}


connected with the presence of air in the experimental ampule-reactor.

Another attractive finding related to the formation of structured carbon deposits at decomposition of polyvinylchloride was observed when the reaction temperature was increased up to $800^{\circ} \mathrm{C}$. There are still two types of deposited carbon, but the morphology is quite different. The first morphological type can be seen in Fig. $5 \mathrm{a}$, d. It looks like dense "sintered" nanofibers of hundreds $\mathrm{nm}$ is diameter, and reminds the carbon product resulted from the decomposition of polytetrafluorethylene (Fig. 2c). The second type is the structured carbon of unique regular shape of microcubes with pyramidal cavities (Fig. 5b, c). The length of the cube edges is $4-5 \mu \mathrm{m}$, and the base of pyramidal cavity is $2 \times 2 \mu \mathrm{m}$. Besides the whole cubes, some incomplete ("broken") fragments are presented. EDX analysis of the cubes has revealed that they contain dominantly carbon (70-85 at.\%) and residual nickel (15-30 at.\%) with traces of chlorine. The origin of observed microcubes, their structural features and the mechanism of their formation remain unclear. All this will be the subject of further detailed studies.

Analogously to other studied substrates, polyvinylchloride was also decomposed under the RAPET conditions without adding the catalyst. The product obtained is represented by "sintered" carbon microspheres of 2-3 $\mu \mathrm{m}$ in size (Fig. 6). These spheres are very close morphologically to the carbon product of polyethylene decomposition at the same temperature $\left(800^{\circ} \mathrm{C}\right)$. Appearance of the microspheres as a product of polyvinylchloride decomposition indicates that formation of nanocubes found in the presence of the bulk nichrome catalyst is not connected directly with the nature of the substrate-precursor. All the carbon structures shown in Fig. 4 thus seem to have the catalytic path involved.

\section{Conclusions}

In the present work, catalytic and non-catalytic decomposition of widely used plastic substrates (polyethylene, polytetrafluoroethylene, and polyvinylchloride) was studied under the RAPET conditions (closed volume and elevated temperatures). Self-disintegration of the bulk $\mathrm{Ni}$-based metallic alloy ( $\mathrm{Ni}-\mathrm{Cr}$ wire) was shown to take place with significant intensity only when polyvinylchloride was used as a substrate. This plastic contains simultaneously hydrogen and halogen atoms required to initiate metal dusting of nichrome. Experiments with polyethylene and polytetrafluoroethylene do not lead to noticeable exposure of the self-disintegration process, even at temperatures as high as $800^{\circ} \mathrm{C}$. Dense carbon deposits was the predominant form of the carbon product resulting from decomposition of these hydrogenonly and halogen-only containing substrates similar to ones described in [12]. At the same time, it should be noted that small amounts of catalytically derived nanofibrous carbon were observed while decomposing the polytetrafluoroethylene at $800^{\circ} \mathrm{C}$.

Decomposition of polyvinylchloride in the presence of nichrome wire is accompanied with intensive selfdisintegration of the latter with subsequent formation of carbon nanofiber in all the studied temperature range $\left(500-800^{\circ} \mathrm{C}\right)$. This result agrees well with the previously made assumption that the self-disintegration process is significantly facilitated if the reaction mixture contains both the hydrogen and the halogen sources $[7,8]$. The mechanism of the observed phenomenon is considered to be schematically described below.

$\mathrm{Ni}+2 \mathrm{HX} \rightarrow \mathrm{NiX}_{2}+\mathrm{H}_{2}$

$\mathrm{NiX}_{2}+\mathrm{H}_{2} \rightarrow \mathrm{Ni}+2 \mathrm{HX}$

where $\mathrm{X}$ is halogen present in the composition of the substrate-precursor.

Due to these reactions, the surface of the bulk metal is loosened, thus facilitating self-dispersion of the alloy with the formation of nickel particles catalyzing the growth of carbon nanofibers.

Besides the mentioned above, formation of unusual solid-phase microcrystals of regular cubic shape with pyramidal cavities on each crystal facet was observed during the catalytic decomposition of polyvinylchloride. While these cubes consist of carbon (70-85 at.\%) and nickel (15-30 at.\%), nichrome self-dispersion is believed to take place under the studied conditions (RAPET at $800^{\circ} \mathrm{C}$ ). The carbon-nickel microcubes obtained are of great attractiveness, and thus will be studied in detail in the nearest future.

In order to conclude, the results of the present research are quite perspective for processing of plastic wastes. The proposed approach is attractive due to its simplicity, single-staging, absence of the catalyst preparation procedures, and, finally, formation of valuable carbon product with unique structure.

Funding This study was funded by the Russian Foundation for Basic Research (Grant Number 16-33-60034).

\section{Compliance with ethical standards}

Conflict of interest The authors declare that they have no conflict of interest. 


\section{References}

1. Tibbetts GG, Lake ML, Strong KL, Rice BP (2007) A review of the fabrication and properties of vapor-grown carbon nanofiber/ polymer composites. Compos Sci Technol 67:1709-1718

2. Feng L, Xie N, Zhong J (2014) Carbon nanofibers and their composites: a review of synthesizing, properties and applications. Materials 7:3919-3945

3. Song H, Shen W (2014) Carbon nanofibers: synthesis and applications. J Nanosci Nanotechnol 14:1799-1810

4. Bauman YI, Mishakov IV, Buyanov RA, Vedyagin AA, Volodin AM (2011) Catalytic properties of massive iron-subgroup metals in dichloroethane decomposition into carbon products. Kinet Catal 52:547-554

5. Bauman YI, Kenzhin RM, Volodin AM, Mishakov IV, Vedyagin AA (2012) Formation of growth centers of carbon nanofibres during self-dispersion of Ni-containing alloys: studies by means of ferromagnetic resonance. Chem Sustain Dev 20:143-155

6. Mishakov IV, Bauman YI, Korneev DV, Vedyagin AA (2013) Metal dusting as a route to produce active catalyst for processing chlorinated hydrocarbons into carbon nanomaterials. Top Catal 56:1026-1032

7. Kenzhin RM, Bauman YI, Volodin AM, Mishakov IV, Vedyagin AA (2016) Structural self-organization of solid-state products during interaction of halogenated compounds with bulk $\mathrm{Ni}-\mathrm{Cr}$ alloy. Mater Lett 179:30-33

8. Kenzhin RM, Bauman YI, Volodin AM, Mishakov IV, Vedyagin AA (2017) Interaction of bulk nickel and nichrome with halogenated butanes. React Kinet Mech Catal 122:1203-1212
9. Pol SV, Pol VG, Gedanken A (2004) Reactions under autogenic pressure at elevated temperature (RAPET) of various alkoxides: formation of metals/metal oxides-carbon core-shell structures. Chem Eur J 10:4467-4473

10. Pol SV, Pol VG, Gedanken A (2005) Novel synthesis of high surface area silicon carbide by RAPET (reactions under autogenic pressure at elevated temperature) of organosilanes. Chem Mater 17:1797-1802

11. Butovsky E, Perelshtein I, Nissan I, Gedanken A (2013) Fabrication, characterization, and printing of conductive ink based on multi core-shell nanoparticles synthesized by RAPET. Adv Funct Mater 23:5794-5799

12. Pol VG (2010) Upcycling: converting waste plastics into paramagnetic, conducting, solid, pure carbon microspheres. Environ Sci Technol 44:4753-4759

13. Mishra N, Das G, Ansaldoc A, Genovese A, Malerba M, Povia M, Ricci D, Fabrizio ED, Zitti ED, Mad Sharon, Mah Sharon (2012) Pyrolysis of waste polypropylene for the synthesis of carbon nanotubes. J Anal Appl Pyrol 94:91-98

14. Bazargan A, McKay G (2012) A review-synthesis of carbon nanotubes from plastic wastes. Chem Eng J 195:377-391

15. Zhuo C, Levendis YA (2014) Upcycling waste plastics into carbon nanomaterials: a review. J Appl Polym Sci 131:39931

16. Nxumalo EN, Coville NJ (2010) Nitrogen doped carbon nanotubes from organometallic compounds: a review. Materials $3: 2141-2171$ 